\title{
АНАЛИЗ ОСНОВНЫХ СУЩЕСТВУЮЩИХ ФАКТОРОВ РИСКА У ПАЦИЕНТОВ С ДИАГНОЗОМ COVID-19 В AMAPÁ, AMAZÔNIA, БРАЗИЛИЯ
}

\section{ОРИГИНАЛЬНАЯ СТАТЬЯ}

OLIVEIRA, Arthur Aires de ${ }^{1}$, MOREIRA, Danilo José Silva², PIMENTEL, Jhon Allyson Sena ${ }^{3}$, COSTA, Pedro Henrique de Magalhães ${ }^{4}$, DIAS, Claudio Alberto Gellis de Mattos $^{5}$, ARAÚJO, Maria Helena Mendonça de ${ }^{6}$, OLIVEIRA, Euzébio de ${ }^{7}$, DENDASCK, Carla Viana ${ }^{8}$, SOUZA, Keulle Oliveira da ${ }^{9}$, FECURY, Amanda Alves ${ }^{10}$

\section{OLIVEIRA, Arthur Aires de. Et al. Анализ основных существующих фракторов} риска у пациентов с диагнозом COVID-19 в Amapá, Amazônia, Бразилия. Revista Científica Multidisciplinar Núcleo do Conhecimento. Год 06, эд. 06, Vol. 17, стр. 56-72. Июнь 2021 года. ISSN: 2448-0959, Ссылка доступа: https://www.nucleodoconhecimento.com.br/здравоохранение/риска-у-пациентов, DOI: 10.32749/nucleodoconhecimento.com.br/ru/90565

\footnotetext{
${ }^{1}$ Медицинский ученый. Федеральный университет Amapá (UNIFAP).

${ }^{2}$ Медицинский ученый. Федеральный университет Amapá (UNIFAP).

${ }^{3}$ Медицинский ученый. Федеральный университет Amapá (UNIFAP).

${ }^{4}$ Медицинский ученый. Федеральный университет Amapá (UNIFAP).

${ }^{5}$ Биолог, кандидат теоретических и летных исследований, профессор и исследователь курса химической степени Института базового, технического и технологического образования Amapá (IFAP) и Высшей программы профессионального и технологического образования (PROFEPT IFAP).

${ }^{6}$ Доктор педагогических и медицинских наук, профессор и исследователь медицинского курса кампуса Масара́, Федеральный университет Amapá (UNIFAP).

${ }^{7}$ Биолог, доктор философии в области тропических болезней, профессор и исследователь курса физкультуры, Федеральный университет Pará (UFPA).

8 Богослов, кандидат психологических наук, исследователь Центра исследований и перспективных исследований (СЕРА).

${ }^{9}$ Социолог, специалист по менеджменту и преподаванию высшего образования, студент магистратуры по антропогенным исследованиям в Amazônia (UFPA).

${ }^{10}$ Биомедицинская, phD в области тропических болезней, профессор и исследователь медицинского курса кампуса Масара́, Федеральный университет Amapá (UNIFAP), проректор по исследованиям и аспирантуре (PROPESPG) Федерального университета Amapá (UNIFAP).
}

RC: 90565

Ссылка доступа: https://www.nucleodoconhecimento.com.br/здравоохранение/риска-упациентов 


\section{СВОДКА}

COVID-19 (Coronavirus Disease 2019) является инфекцией, вызванной вирусом SARS-CoV-2, первые случаи заболевания были зарегистрированы в декабре 2019 года в городе Wuhan. Было установлено, что у пациентов с сопутствующими инфекциями существует более высокий риск осложнений и смертности в случае инфицирования. Эта болезнь была рассмотрена Всемирной организацией здравоохранения в 2020 году как чрезвычайная ситуация в области общественного здравоохранения, а в Бразилии министерство здравоохранения осуществило меры по эпидемиологическому мониторингу случаев заболевания, зарегистрированных в различных федеральных подразделениях страны. Среди них Амапа предполагает высокий уровень заболеваемости и смертности. Это исследование направлено на анализ основных предыдущих фракторов риска, обнаруженных у пациентов с COVID-19 в штате Amapá. Для этого вторичные данные, имеющиеся в группе коронавируса Amapá, были использованы в ходе поиска, проведенного 22 мая 2020 года. На платформе были собраны данные о количестве пациентов с диагнозом COVID-19, а также о количестве смертей от этой болезни, при этом была проведена совместная оценка по каждой теме сопутствующих заболеваний. После группирования этих данных в электронные таблицы Microsoft Excel был проведен количественный и описательный анализ этих данных. В штате до дня сбора было зарегистрировано в общей сложности 28927 пациентов с диагнозом COVID-19. Около 1,31\% (378 случаев) из них имели фрактор риска, который может восприимчивы их к развитию осложнений в результате COVID-19, с диабетом и хроническими заболеваниями сердца условия с наибольшим количеством записей. Тем не менее в штате Amapá было обнаружено 420 случаев смерти у пациентов с COVID-19. Из этого общего числа 75,71\% (318 случаев) имели предыдущие факторы риска более высокого риска осложнений из-за COVID-19, при этом гипертония и диабет были преобладающими состояниями. В ходе исследования было замечено в Amapá, что значительная часть населения пациентов с диагнозом болезни и кто умер, имели историю фрактора риска для COVID-19. В связи с уведомлением о 
факторах риска в смертельной ситуации, которые не были зарегистрированы в количестве диагностированных пациентов, а также неспецифической степени, присущей состоянию пациентов, контролируемых государственными механизмами здравоохранения, не удалось провести более детальный анализ степени осложнений и летальности, порожденных данным фрактором риска и его связью с коронавирусной инфекцией.

Ключевые слова: COVID-19, Факторы риска, Коморбидность.

\section{ЗНАКОМСТВО}

В первой половине декабря 2019 года первый случай пневмонии неизвестного происхождения был зарегистрирован в Wuhan, провинция Hubei, China. Впоследствии, вспышка тяжелой острой пневмонии респираторного синдрома (SRAG) преследует провинцию, привлекая внимание Всемирной организации здравоохранения (ВО3), которая, начиная с обрезания его присвоения, заявил, что ситуация была эпидемия, вызванная новым подтипом вируса, принадлежащего к семейство coronaviridae, коронавирус-2, составляющих чрезвычайной ситуации общественного здравоохранения международного значения (ESPII), января 30, 2020 (COSTA et al., 2020a; FERRARI, 2020; GAUTRET et al., 2020; GUAN et al., 2020; LI et al., 2020; LIMA et al., 2020; MARTINSCHAVES; GOMES; GOMEZ, 2020; OLIVEIRA et al., 2020; RENU; PRASANNA; GOPALAKRISHNAN, 2020; RODRÍGUEZ-COLA et al., 2020; TAY et al., 2020; ZHAI et al., 2020).

В фреврале 2020 года Генеральный директор ВОЗ назвал болезнь как болезнь коронавируса-19 (COVID-19), с инфекцией, вызванной SARS-CoV-2 (выражением на английском языке: Severe Acute Respiratory Syndrome Coronavirus 2), один из семи подтипов известных коронавирусов (COSTA et al., 2020a; RENU; PRASANNA; GOPALAKRISHNAN, 2020; HONG et al., 2020).

C бразильской точки зрения, инфекция COVID-19 была объявлена Министерством здравоохранения (MS) как чрезвычайная ситуация в области RC: 90565

Ссылка доступа: https://www.nucleodoconhecimento.com.br/здравоохранение/риска-упациентов 
общественного здравоохранения национального значения (ESPIN) 3 февраля 2020 года, и ее первый случай был подтвержден в Бразилии 26 февраля (OLIVEIRA et al., 2020; OLIVEIRA; LUCAS; IQUIAPAZA, 2020). В качестве меры поддержки системе здравоохранения Департамент информатики Единой системы здравоохранения (DATASUS) в рамках Плана действий на случай чрезвычайных ситуаций принял меры по оказанию виртуальной помощи населению через приложение "Coronavírus SUS" (BRASIL, 2020a).

В Бразилии прогресс в борьбе с пандемией в основном контролируется MS c помощью статистических индексов, отражающих состояние каждой фредеративной единицы (STATE), а штат Амапа предполагает высокие эпидемиологические индексы, особенно в том, что касается коэффрициента заболеваемости COVID-19 (BRASIL, 2020c).

До 17 июля, В 2020 году Аmaра́ возглавил бразильский Север в рейтинге коэффициента заболеваемости COVID-19 на уведомление STATE (число подтвержденных случаев на 100000 жителей), стоимость которого составила 3637,4 , за которым следует Рорайма, с 3327,1, что, в свою очередь, было подтверждено штатом Амазонка, с 1962,0. Что касается коэфффициента смертности COVID-19 (число смертей на 100000 жителей), то Amapá был на 7-м месте $(55,2)$ (BRASIL, 2020c).

Факторы риска связаны с любыми условиями, которые ухудшают иммунный ответ организма на болезнь. Таким образом, эти фракторы включают как сопутствующие заболевания, то есть хронические заболевания, ранее установленные и которые действуют одновременно в организме пациента, а также особенности отдельных лиц, а именно: puerperal состояние, курение, потребление алкоголя, среди других (PIRES; CARVALHO; XAVIER, 2020; ENGIN; ENGIN; ENGIN, 2020; KHALIQ; PHOSWA, 2020).

Исследования показывают, что пациенты, инфицированные COVID-19, которые имеют сопутствующие заболевания имеют более высокую иммунологическую 
уязвимость, а также более высокий уровень летальности по сравнению с пациентами, пострадавшими только от вируса. Основные сопутствующие заболевания перечислены включают гипертонию, диабет, болезни сердца и хронические заболевания дыхательной системы (COSTA et al., 2020a; FERRARI, 2020; MARTINS-CHAVES; GOMES; GOMEZ, 2020; RENU; PRASANNA; GOPALAKRISHNAN, 2020; YANG et al., 2020).

Учитывая глобальную панораму и с учетом состояния государства Аmapá, необходимо проводить анализы, исследования и продвижение по службе в научных исследованиях, с тем чтобы установить надлежащие методы управления для пациентов, затронутых основными заболеваниями и/или состояниями, которые представляют потенциальные фракторы риска для прогноза пациентов с диагнозом ТОРС-КОВ-2, с целью смягчения осложнений, представленных ими (RENU; PRASANNA; GOPALAKRISHNAN, 2020; YANG et al., 2020).

\section{ЦЕЛЕЙ}

Проанализировать распределение основных уже существующих фракторов риска у пациентов с диагнозом COVID-19 в штате Amapá, Амазонка, Бразилия.

\section{МЕТОДОЛОГИИ}

Это исследование было направлено на количественный и описательный анализ числа пациентов, затронутых COVID-19 в Amapá, которые свидетельствуют о фракторах риска заболевания, и для этого, консультации были сделаны 22 мая 2020 года в Коронавирус Amapá Группа (painel.corona.ap.gov.br).

На главной странице панели Coronavirus Amapá была нажата кнопка «загрузить CSV», чтобы получить основные данные о случаях COVID-19 в штате Amapá. Затем в поле «Подтвержденные сопутствующие заболевания пациентов» на той же странице была выбрана опция «Inspect» в правом верхнем углу, чтобы создать список сопутствующих заболеваний и их числовую взаимосвязь. Тот же 
процесс был повторен в блоке «Сопутствующие заболевания у пациентов со смертью». Впоследствии для каждого из полученных списков в поле «Fromatted CSV» была выбрана опция «Download CSV», чтобы получить фрайлы и использовать их в Microsoft Excel.

В этих файлах некоторые сопутствующие и риск-ситуации были объединены в один и тот же набор для стандартизации результатов. Быть: они сгруппированы - в хронических болезней сердца - хронические болезни сердца, хронические болезни сердца, хронические болезни сердца, хронические заболевания сердца, сердечно-сосудистые заболевания, болезни сердца, кардиопатические заболевания, хронические болезни сердца, болезни сердца; также в Хронические респираторные заболевания - хронические респираторные заболевания, хронические респираторные заболевания, хронические респираторные заболевания, хронические декомпенсированные хронические респираторные заболевания; Они все еще находятся в - Гипертония-гипертония, HAS, гипертонический; снова в - болезни сердца и гипертония - Хронические болезни сердца (HAS), хронические болезни сердца - HAS, сердечно-сосудистые заболевания, включая гипертонию, хронические болезни сердца HAS, болезни сердца (HAS), хронические болезни сердца - HAS; а также в - Иммуносупрессияиммуносупрессия, хромосомные заболевания перевозчика или состояние FR; Снова в - Болезни сердца и диабет - хронические болезни сердца и диабета, сердца и диабета; Таким образом, они находятся в высокой опасности беременности - высокого риска беременных женщин, высокого риска беременных женщин; Наконец, в - Расширенный этап хронических заболеваний почек - Расширенный этап хронических заболеваний почек, Передовые стадии хронических заболеваний почек, Передовые стадии хронических заболеваний почек.

В таблице "Коморбидность пациентов В смерти" число случаев, зарегистрированных как "Нет информации", было проигнорировано из-за безрезультатности этого отсечения. Другие данные были отобраны и сгруппированы, как описано выше. 
В настоящем исследовании использовались вторичные данные, имеющиеся в общественном доступе, и учитываются этические аспекты, основанные на резолюции 466/12.

\section{РЕЗУЛЬТАТЫ}

Среди 28927 подтвержденных случаев COVID-19 в Amapá, которые были зарегистрированы до дня сбора, приблизительно 1,31\% пациентов (378 случаев) имели фрактор риска, который мог бы подвержены их развитию осложнений из-за участия ТОРС-КоВ-2, как показано на рисунке 1.

Рисунок 1 - Наличие факторов риска у пациентов с диагнозом COVID-19 в Amapá до 22 мая 2020 года.

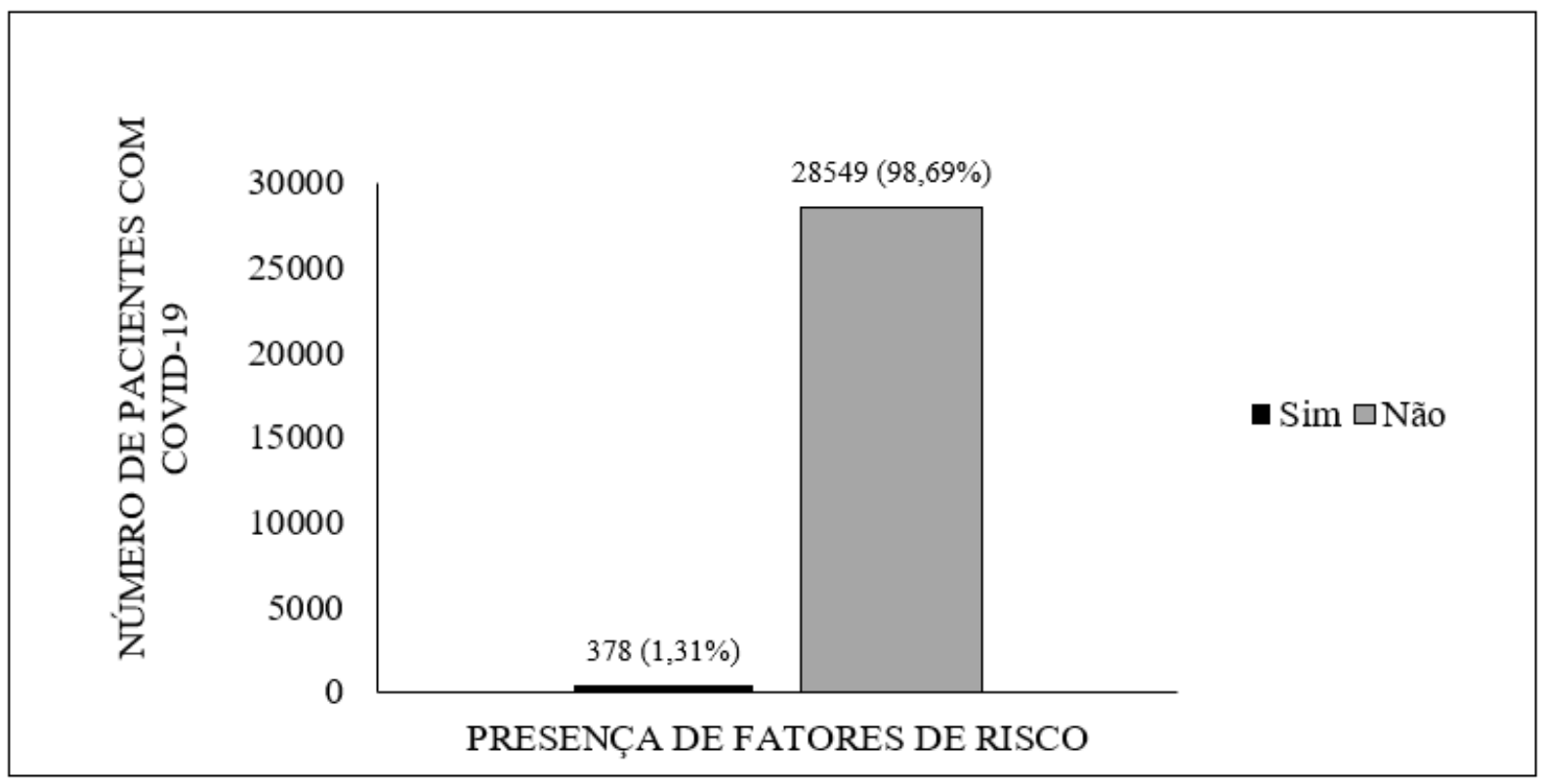

Источник: Коронавирусная группа Aтара́.

Что касается пациентов с фракторами риска, то 5,02\% из них (19 случаев) имели более одного случая. Ассоциации включают болезни сердца и гипертонию $(3,44 \%)$, болезни сердца и диабет $(1,06 \%)$, ангиопластику и коронарный атероматоз (0,26\%) хронические заболевания почек и диабет $(0,26 \%)$. 
Что касается основных фракторов риска, о которых сообщили только пострадавшие пациенты, то было установлено, что большинство из них имели диабет 29,89\% (112 случаев), за которыми следовали хронические заболевания сердца с $28,84 \%$ (109 случаев).

Отмечается, что заболевания кардиологической сферы присутствуют в большинстве случаев, учитывая, что 173 пациента в общей сложности страдают от заболеваний этого класса, добавляя людей с множественными факторами риска и лиц только с сердечными заболеваниями. 
Таблица 1 - Распределение фракторов риска у пациентов с диагнозом COVID-19 B Amapá.

\begin{tabular}{|c|c|c|}
\hline Факторы риска & $\begin{array}{l}\text { Количество } \\
\text { отчетов }\end{array}$ & $\begin{array}{l}\text { Процент } \\
\text { (\%) }\end{array}$ \\
\hline диабет & 113 & $29,89 \%$ \\
\hline Хронические болезни сердца & 109 & $28,84 \%$ \\
\hline $\begin{array}{l}\text { Хронические } \quad \text { респираторные } \\
\text { заболевания }\end{array}$ & 71 & $18,78 \%$ \\
\hline гипертония & 46 & $12,17 \%$ \\
\hline Болезни сердца и гипертония & 13 & $3,44 \%$ \\
\hline иммуносупрессия & 11 & $2,91 \%$ \\
\hline Болезни сердца и диабет & 4 & $1,06 \%$ \\
\hline Беременность высокого риска & 4 & $1,06 \%$ \\
\hline $\begin{array}{l}\text { Прогрессная стадия } \quad \text { хронических } \\
\text { заболеваний }\end{array}$ & 3 & $0,79 \%$ \\
\hline Ангиопластика и коронарный атероматоз & 1 & $0,26 \%$ \\
\hline Хронические болезни сердца и диабет & 1 & $0,26 \%$ \\
\hline Цирроз печени & 1 & $0,26 \%$ \\
\hline депрессия & 1 & $0,26 \%$ \\
\hline Общая & 378 & $100,00 \%$ \\
\hline
\end{tabular}

Источник: Коронавирусная группа Aтара́.

Всего на момент исследования было зарегистрировано 420 случаев смерти. Из этого количественного, было установлено, что 318 пациентов имели историю сопутствующих заболеваний или предрасполагающие состояние осложнений. 
Рисунок 2 - Наличие факторов риска у пациентов, которые умерли с COVID-19 в Amapá до 22 мая 2020 года.

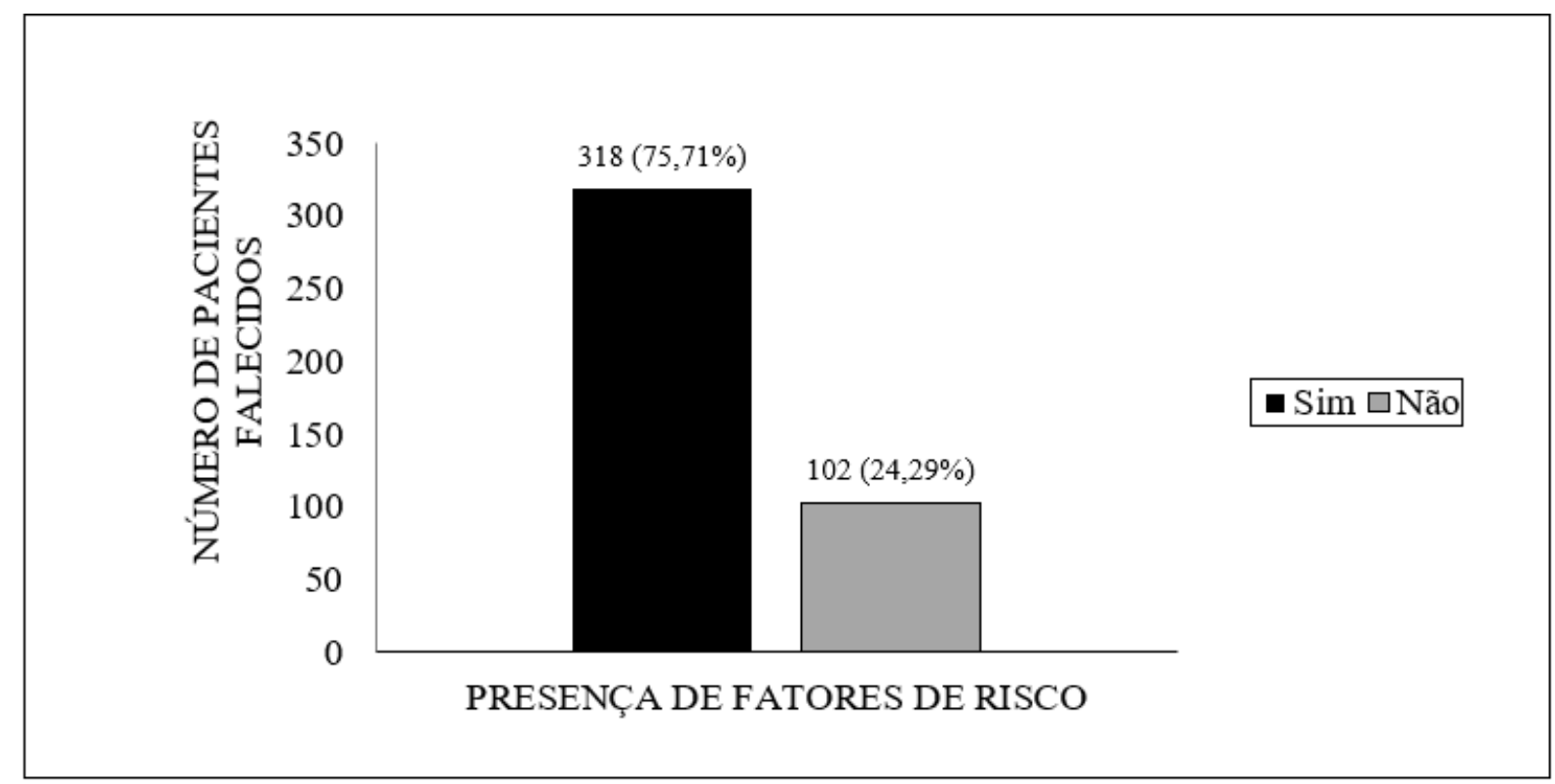

Источник: Коронавирусная группа Amaра́.

Что касается фракторов риска, присутствующих у пациентов, которые умерли, основной рекорд гипертонии, присутствует в 38,68\% пациентов (123 случаев). Было также отмечено наличие других отягчающих факторов, которые ранее не были описаны у лиц с подтвержденной инфекцией. Это ожирение, цереброваскулярные заболевания, неоплазия, курение, болезнь Альцгеймера, заболевания поджелудочной железы, денге, употребление алкоголя и puerperal период. 
Таблица 2 - Распределение фракторов риска, зарегистрированных у пациентов, которые умерли с COVID-19 в Amapá.

\begin{tabular}{|l|c|c|}
\hline коморбидность & Число смертей & Процент (\%) \\
\hline гипертония & 123 & $38,68 \%$ \\
\hline диабет & 93 & $29,25 \%$ \\
\hline Болезни кожи & 27 & $8,49 \%$ \\
\hline сердце & 19 & $5,97 \%$ \\
\hline ожирение & 19 & $5,97 \%$ \\
\hline Респираторные заболевания & 15 & $4,72 \%$ \\
\hline Цереброваскулярные заболевания & 8 & $2,52 \%$ \\
\hline Иммуносупрессивные заболевания & 3 & $0,94 \%$ \\
\hline Новообразования & 3 & $0,94 \%$ \\
\hline курение & 2 & $0,63 \%$ \\
\hline Болезнь Альцгейера & 1 & $0,31 \%$ \\
\hline Заболевания поджелудочной железы & 1 & $0,31 \%$ \\
\hline Заболевания печени & 1 & $0,31 \%$ \\
\hline лихорадка денге & 1 & $0,31 \%$ \\
\hline Этилист & 1 & $0,31 \%$ \\
\hline родильница & 1 & $0,31 \%$ \\
\hline Общая & 318 & $\mathbf{1 0 0 , 0 0 \%}$ \\
\hline
\end{tabular}

Источник: Коронавирусная группа Aтара́

\section{ОБСУЖДЕНИЯ}

В штатах региона Амазонки Бразилии наблюдаются значительные социальноэкономические расхождения по сравнению с другими, что может отражать наибольшее воздействие на наблюдаемую систему здравоохранения. В штате Amapá, после того, как 25 марта 2020 года было зарегистрировано первое случае, COVID-19 распространился массово, как и в других федеративных подразделениях (DIAS et al., 2020; MENDONÇA et al., 2020). 
Число пациентов с COVID-19, которые имеют фракторы риска заболевания в Amapá, по сравнению с общим числом инфицированных пациентов, считается низким. В литературе существует консенсус В отношении того, что сопутствующие заболевания и ситуации иммунологической уязвимости считаются отягчающими фракторами для состояния COVID-19, и этот фракт, возможно, побудил людей из групп риска удвоить свою помощь (COSTA et al., 2020b; RODRÍGUEZ-COLA et al., 2020).

В исследовании, проведенном Wang et al. (2020 г.), было сообщено, что из 36 пациентов, нуждахся в медицинской помощи в отделении интенсивной терапии (UTI), 26 (72,2\%) сопутствующих заболеваний, что свидетельствует о том, что история хронических заболеваний может привести к развитию осложнений. Silva et al. (2020), в клиническом и эпидемиологическом исследовании характеристики с 1560 пациентов с диагнозом COVID-19 из города Масара́-AP, обнаружили, что 102 пациентов (6,54\%) сопутствующих заболеваний.

Основными сопутствующими заболеваниями, зарегистрированными только у пациентов в настоящем исследовании, были диабет, хронические заболевания сердца, хронические респираторные заболевания и гипертония. Мета-анализ, сделанный в общей сложности 1576 участников Yang et al. (2020) также выделяет гипертонию $(21,1 \%)$, диабет $(9,7 \%)$, сердечно-сосудистые заболевания $(8,4 \%)$ и заболевания дыхательной системы (1,5\%) как наиболее частые сопутствующие заболевания. Silva et al. (2020) установлено, что из 102 пациентов с сопутствующими заболеваниями с диагнозом COVID-19 оценивается, хронические сердечно-сосудистые заболевания $(38,33 \%)$, диабет $(24,16 \%)$ хронических респираторных заболеваний $(13,33 \%)$ наиболее распространенными факторами риска. Эти условия связаны с высоким риском серьезных проявлений COVID-19 (GOMES; GOMEZ, 2020; MARTINS-CHAVES), который объясняется по частям повышенной экспрессией фрермента ангиотензина-преобразовательа 2 (ACE2) в альвеолярных, сердечных, $\beta$ поджелудочной и сосудистой эпителиальных клетках эндотелия. ЕСА2 позволяет входом вируса в клетки организма, что подтверждает отказ 
соответствующих органов во время инфекционного заболевания (ANGHEBEM; REGO; PICHETH, 2020; ASKIN; TANRIVERDI; ASKIN, 2020).

Некоторые пациенты также имели другие сопутствующие заболевания и фракторы риска, включая иммуносупрессию, беременность высокого риска, хронические заболевания почек, ангиопластику с коронарным атеросклерозом, цирроз печени и депрессию.

Данные настоящего исследования показывают летальность для пациентов с иммуносупрессивными заболеваниями 27,27\%. Считается, что чем больше повышается иммунитет пациентов с COVID-19, тем хуже прогноз заболевания, причина, по которой иммунодепрессированные пациенты являются группами риска заболевания (GOMES; GOMEZ, 2020; MARTINS-CHAVES). Тем не менее, есть доклад в литературе, что большинство пациентов с иммунодепрессией оценивается следуют с хорошим прогнозом. Исследование, проведенное Hrusak et al. (2020) с педиатрическими пациентами, проходящими противораковое лечение, показали, что из 9 детей с диагнозом COVID-19, 8 с легкими симптомами или даже аймптоматические. Кроме того, Bussalino et al. (2020) засвиделение, что поддержание иммуносупрессии может сделать течение COVID-19 мягким для пациента за счет уменьшения бури цитокинов, характерных для заболевания.

Что касается беременности, то В штате зарегистрировано 4 случая беременности высокого риска. Материнские риски подозреваются, потому что системные и местные иммунологические изменения вызваны в организме матери, начиная от провоспалительным состоянием до противовоспалительного состояния (LIU et al., 2020). В случае беременных женщин с COVID-19, метаанализ, сделанный Trippela et al. (2020) c 275 беременных женщин, пострадавших от этой болезни показали, что 269 описали их симптоматики, и 91,82\% были симптомы, в том числе лихорадка и кашель, как наиболее распространенные, и 8,18\% были аймптоматические. Большинство пациентов 
имеют легкие или аимптоматические симптомы, с несколькими сообщениями о развитии осложнений (CAPARROS-GONZALEZ, 2020; TRIPPELA et al., 2020).

Что касается хронических коронарных заболеваний, то в систему эпидемиологического эпиднадзора коронавируса амапа поступило лишь 3 подтвержденных случая заболевания. Тем не менее, 27 случаев смерти пациентов, которые ранее имели сопутствующие заболевания этого класса заболеваний были введены в систему, что свидетельствует о возможном занижении сопутствующих заболеваний. В перспективном когортное исследование, произведенное Cheng et al. (2020) с 701 лиц, пострадавших от COVID-19, было замечено, что 26,7\% людей, проанализированных были гематурии и 43,9\% протеинурии. Эти клинические результаты свидетельствуют о наличии лабораторных проблем (MOITINHO et al., 2020). С учетом того, что SARS-CoV-2 действует непосредственно на приемник ECA2 (ASKIN; TANRIVERDI; ASKIN, 2020) и который уже был оказано переэкспрессии этого фермента в проксимальных трубчатых клеток пациентов с хроническими заболеваниями полости рта (FAN et al., 2020), считается, что история болезней жизни может усугубить картину COVID-19 (RENU; PRASANNA; GOPALAKRISHNAN, 2020). Кроме того, он был связан с предсуществовательским заболеванием почек с нарушением функциональности иммунной системы пациента, процесс, который может быть объяснен воспалительным процессом, установленным во время нефропатии. Это способствует идее, что эти заболевания являются фракторами риска для COVID19 (OYELADE; ALQAHTANI; CANCIANI, 2020).

Что касается взаимосвязи между ангиопластикой и коронарным атероматом с COVID-19, то в литературе не было обнаружено никаких сообщений, которые могли бы поддерживать связь между этими фракторами риска и вирусной инфекцией.

Из зарегистрированных случаев заболевания печени в штате Amapá зарегистрирован лишь один случай цирроза печени. Также была зафиксирована 
смерть пациента, который ранее был заболеванием печени, но невозможно утверждать, что это тот же пациент, так как уже существующие заболевания не были указаны. Литература показывает, что инфекция SARS-CoV-2 также связана c печенью (AGHAGOLI et al., 2020), и наличие заболеваний печени считается определяющим фрактором тяжести картины COVID-19 (RENU; PRASANNA; GOPALAKRISHNAN, 2020). Мета-анализ Oyelade, Alqahtani и Canciani (2020) с участием 5595 пациентов показали распространенность заболеваний печени у пациента с COVID-19 3\%, и из них, тяжесть была зарегистрирована в 57,33\%. То же исследование показало, уровень смертности 17,65\%. Несмотря на тяжесть, в литературе не было никакой корреляции между уже существующим заболеванием печени и результатом COVID-19.

В единственном случае депрессии, зарегистрированном в государстве, научное сообщество обеспокоено возможностью развития нейропсихических заболеваний, также составляющих риск заболевания COVID-19. Психические расстройства, а также состояние психологического расстройства, непосредственно влияют на фрункционирование иммунной системы человека, которая может восприимчивы к вирусным инфекциям дыхательных путей, что свидетельствует о возможной связи между эмоциональным состоянием пациента и степенью вовлеченности SARS-CoV-2 (RAJKUMAR, 2020). Однако воздействие вируса на пациентов, о которых идет речь, а также иммунологическая реакция, генерируемая организмом, не очень хорошо выяснены в литературе (TROYER; KOHN; HONG, 2020). Для пациентов с историей слабости психического здоровья, больше внимания следует уделять состояние, так как это может рецидив или ухудшиться в условиях установленного сценария пандемии и возможной диагностики COVID-19 (YAO; CHEN; XU, 2020).

Данные, собранные с коронавирусной панели Аmapá, свидетельствуют о том, что $76 \%$ пациентов, которые умерли, имели сопутствующие заболевания или ранее существующие отягчающие фракторы. Эпидемиологическое обследование, проведенное Almeida et al. (2020) в штате Maranhão обнаружили, что из 100 пациентов с диагнозом COVID-19 и которые умерли, 78\% имели 
предыдущие сопутствующие заболевания, предполагая, что картина похожа на то, что наблюдалось в Amapá.

Отмечается, что гипертония является наиболее зарегистрированным фактором риска среди пациентов с амипансе, которые умерли. Однако определить уровень летальности для гипертонийных больных невозможно, так как число подтвержденных случаев заболевания ниже, чем зарегистрированных о смерти. Тем не менее, литература показывает, что это заболевание является одним из основных отягчающих факторов для состояния COVID-19, наряду с другими сердечно-сосудистыми заболеваниями (AGHAGOLI et al., 2020; ALMEIDA et al., 2020; FERRARI, 2020). Патофизиологический механизм, предложенный для объяснения этой взаимосвязи, включает в себя систему реиттина. Из-за более высокой привязки SARS-CoV-2 к ECA2, концентрация этого фермента снижается в хозяине, что приводит к высокому уровню ангиотензина II и снижению ангиотензина 1-7, эффректы, которые генерируют сосудосуживающее и удержание натрия и ухудшают гипертонический состояние (TADIC et al., 2020).

Что касается взаимосвязи между диабетом и COVID-19, можно ухудшить клинические условия пациента, учитывая, что SARS-COV-2 способствует повышению уровня глюкозы в крови у пациентов с диабетом 2 типа (BORNSTEIN и др., 2020). Ухудшение состояния диабетиков может быть связано с тем, что эта сопутствующие заболевания снижает естественный иммунитет организма (COSTA et al., 2020b; MA; HOLT, 2020). Что касается зарегистрированных случаев этого заболевания в Амапа, то заметно, что число подтвержденных пациентов (112) количественно близко к тем, кто умер (93), тем самым подтверждая идею о том, что диабет может усугубить состояние covid-19 до такой степени, что пациент умирает (COSTA et al., 2020b; RONCON et al., 2020).

Другой важной характеристикой диабета и отношения SARS-COV-2 являются механизмы осложнений в результате союза этих двух фракторов, поскольку лица, которые имеют диабет и инфицированы вирусом, более рискуют развития осложнений (LACOBELLIS, 2020). Гипотеза о том, что диабет действует 
негативно, вместе с вирусом, связана с ECA2, так как он присутствует в нескольких тканях и действует как шлюз к инфекционным агентам на поверхности клеток (AGHAGOLI et al., 2020; MA; HOLT, 2020). Кроме того, пациенты, которые используют ингибиторы ангиотензина-подавления ферментов (ACEI), с большей вероятностью развивают осложнения из-за более высокого производства ACE2, что способствует действию SARS-COV-2 (FANG; KARAKIULAKIS; ROTH, 2020; FERRARI, 2020; MA; HOLT, 2020).

Сердечные заболевания, в свою очередь, являются условиями, связанными с худшими прогнозами и высокими показателями летальности (AGHAGOLI et al, 2020). Huang, Wang e Li (2020) показал, что из 41 пациентов с COVID-19 оценивается, 6 (15\%) были заболевания сердечной сферы. В самом деле, существует значительное число пациентов с сердечными заболеваниями, которые умерли в Amapá (19), генерации смертности 15,07\%. Литература связывает эту тяжесть с наличием в сердце рецепторов ЕСА2, которые высоко выражены в органе, что может привести к развитию острой травмы миокарда и хронического сердечно-сосудистого повреждения (AGHAGOLI et al, 2020). Кроме того, преклонный возраст и эффрект иммуносенсции наблюдается у большинства пациентов с сердечными заболеваниями могут быть связаны с прогнозом состояния COVID-19 (DRIGGIN et al., 2020).

Было также отмечено значительное число пациентов с респираторными заболеваниями, что соответствует летальности $21,12 \%$ с учетом числа пациентов с диагнозом COVID-19, которые сообщили о уже существующих сопутствующих заболеваниях. Предварительное наличие респираторных заболеваний связано с тяжелыми состояниями COVID-19 (MARTINS-CHAVE; GOMES; GOMEZ, 2020; YANG et al., 2020). Известно, что иммунная система реагирует на инфекцию SARS-CoV-2 воспалительным процессом, отмеченным действием иммунных клеток, некрозом тканей и местной гиперплазии, эффектами, которые могут ухудшить процесс газопроменности легких и создать картину тяжелой пневмонии (GIMENEZ et al., 2020), что может оправдать, почему респираторные заболевания являются фрактором риска для COVID-19. 
Кроме того, в информации, представленной коронавирусом Amapá Группа, отмечается, что некоторые фракторы риска для COVID-19, найденные у пациентов, которые умерли, не были зарегистрированы из сессии фракторов риска пациентов с диагнозом болезни, такие как ожирение, цереброваскулярные заболевания, неоплазмы, курение, болезнь Альцгеймера, заболевания поджелудочной железы, денге, алкогольный изм и puerperal период. Это препятствует надежному анализу эпидемиологического сценария местности. Тем не менее, некоторые аспекты этих условий были обсуждены в литературе.

О ожирении, давно известно, что это непосредственно связано с плохими прогнозами и трудоемкого восстановления в случаях вирусных инфекций. Этот вывод объясняется постоянным воспалительным состоянием, характерным для людей с ожирением, поддерживаемым высоким уровнем воспалительных препаратов, что способствует ухудшению иммунологической реакции, генерируемой этими пациентами, и, следовательно, снижению способности бороться с инфекционными агентами (COSTA et al., 2020b; HUSSAIN et al., 2020; KORAKAS et al., 2020). В контексте COVID-19, другие фракторы, связанные с ожирением может быть причиной более высоких шансов на ухудшение клинической картины, которая включает в себя дефицит дыхательной механики, низкий уровень обмена газа в легких, повышение устойчивости дыхательных путей, снижение силы легких и более низкий объем легких (STEFAN et al., 2020). Мета-анализ, проведенный Hussain et al. (2020) показал, что из 2451 пациента с индексом массы тела (IMC) более 25 кг/м2 проанализированы, 531 умер, что соответствует летальности 21,66\%. В том же исследовании оценивались пациенты с IMC менее 25к/м2,что составило 24 506. Из них 1701 умер, уровень летальности составил 6,94\%. Сравнение этих значений показывает, что избыточный вес и ожирение могут влиять на прогноз пациента.

Цереброваскулярные заболевания были зарегистрированы в более тяжелых случаях COVID-19 (WANG et al., 2020). Механизм повышения тяжести для этого класса пациентов до сих пор не определен, хотя уже предполагается, что он похож на сердечно-сосудистые заболевания (PRANATA et al., 2020). 
Что касается взаимосвязи между неоплазмами и COVID-19, считается, что онкологические больные подвержены развитию осложнений из-за ослабления их иммунологической активности (ASOKAN; RABADIA; YANG, 2020; AL-QUTEIMAT; AMER, 2020). В самом деле, ретроспективное исследование Zhang et al. (2020) показал, что из 28 оцениваемых онкологических больных 8 умерли (коэффрициент смертности 28,6\%), что свидетельствует о плохом прогнозе для пациентов с допостоямия неоплазм.

История курения, в свою очередь, предполагается в качестве важного фактора риска для развития тяжелых условий заболевания, так как курение связано с нарушением здоровья легких (VARDAVAS; NIKITARA, 2020). Хотя только один случай курения был зарегистрирован у пациентов, которые умерли в нашем эпидемиологическом анализе, Есть исследования, которые демонстрируют повышенный риск тяжести у пациентов с историей курения. Alqahtani et al. (2020), в мета-анализе с участием 139 нынешних курильщиков и 28 бывших курильщиков с диагнозом COVID-19, обнаружили, что 31 текущий курильщиков (22,30\%) и 13 бывших курильщиков (46\%) ухудшилось их клинические условия. В том же исследовании, 5 нынешних курильщиков из 13, которые разработали осложнений умер (смертность 38,5\%). Эти замечания лишь усиливают, что лица, которые уже курили или курили, должны избегать этой практики в разгар пандемии и всегда принимать превентивные меры.

О болезни Альцгеймера, связь между преклонным возрастом и деменцией, замечательные характеристики болезни, а также возможные нарушения нейрокогнитивного развития этих людей во время социальной изоляции в пандемии, поощрять научное сообщество для оценки до существования болезни Альцгеймера в качестве фрактора риска для COVID-19 (BRASIL, 2020b; FERINISTRAMBI; SALSONE, 2020). В случае смертности, Bianchetti et al. (2020) установлено, что из 82 пациентов с диагнозом COVID-19 и с деменцией, 51 $(62,2 \%)$ умер, что выше, чем у $26,2 \%$ наблюдается у пациентов, которые не имеют слабоумия, что указывает на то, что не только болезнь Альцгеймера, но и другие деменции могут быть связаны с тяжелой ковид-19 состоянии. 
Сообщенный случай пациента этилиста который умер предлагает привычку как по возможности фрактор риска для заболевания. Эта ассоциация поддерживается предыдущим знанием, что злоупотребление алкоголем может поставить под угрозу функцию печени (EHRMANN; URBAN; DVORAN, 2019), что напугало бы пациента от осложнений и, как следствие, более тяжелой картины COVID-19.

Взаимосвязь между puerperium и COVID-19 не так хорошо установлена по сравнению с беременностью (AN et al., 2020). Между тем, Tutiya et al. (2020) зарегистрировано два случая образования легочного микротромби у женщин с диагнозом COVID-19. Кроме того, An et al. (2020) сообщили о двух случаях puerperal женщин, которые представили дыхательной недостаточности и ухудшение состояния после родов. Эти наблюдения усиливают необходимость мониторинга состояния пациентов после родов до стабилизации.

Денге и заболевания поджелудочной железы, которые были зарегистрированы в смерти от COVID-19 в Amapá, однако, не было найдено никаких сообщений в литературе о связи этих заболеваний и развития осложнений вирусной инфекции, хотя заболевания, которые компрометации иммунитета пациента, как правило, увеличивают шансы на ухудшение уже существующих условий (MARTINS-CHAVES; GOMES; GOMEZ, 2020).

\section{ЗАКЛЮЧЕНИЕ}

Предварительное наличие сопутствующих заболеваний или других состояний, которые ухудшают иммунный ответ организма является потенциальным отягчающим фрактором инфекционного заболевания, генерируемого новым коронавирусом, который может привести пациента к смерти. В штате Amapá значительная часть пациентов с диагнозом заболевания, которые умерли, в течение многих лет имели фрактор риска для COVID-19.

Основными сопутствующими заболеваниями у пациентов с диагнозом диабет, хронические заболевания сердца, хронические респираторные заболевания и RC: 90565

Ссылка доступа: https://www.nucleodoconhecimento.com.br/здравоохранение/риска-упациентов 
гипертония. Сопутствующим заболеванием с самым высоким показателем в случаях смерти была гипертония.

Уведомление о фракторах риска в диаграмме смерти, которые не были зарегистрированы в картине диагностированных пациентов, а также неспецифическая неспецифическая степень, присущая состоянию пациентов, контролируемых механизмами здравоохранения государства, сделали более подробный анализ степени осложнений и летальности, порожденных данной сопутствующих заболеваний, когда она была связана с коронавирусной инфекцией.

Для большей полноты и надежности результатов такого исследования необходимо получить достаточно точные и согласованные данные, с тем чтобы можно было проанализировать и затем включить пациентов через сеть здравоохранения в научные исследования, что позволит понять и противостоять нынешней пандемии и ее последствиям в Amapá.

\section{ссылки}

AGHAGOLI, G. et al. Cardiac involvement in COVID-19 patients: Risk factors, predictors, and complications: A review. J Card Sug., v. 35, p. 1302-1305, abr. 2020.

ALMEIDA, J. S. et al. Caracterização epidemiológica dos casos de covid-19 no maranhão: uma breve análise. Revista Prevenção de Infecção e Sáude, v. 6, mai. 2020.

AL-QUTEIMAT, O. M.; AMER, A. M. The Impact of the COVID-19 Pandemic on Cancer Patients. Am J Clin Oncol., abr. 2020. DOI: 10.1097/COC.0000000000000712.

ALQAHTANI, J. S. et al. Prevalence, Severity and Mortality associated with COPD and Smoking in patients with COVID-19: A Rapid Systematic Review and Meta-Analysis. PLoS One, v. 15, n. 5, e0233147, mai. 2020. 
AN, P. et al. Postpartum exacerbation of antenatal COVID-19 pneumonia in 3 women. CMAJ, v. 192, n. 22, p. 603-606, jun. 2020.

ANGHEBEM, M. I.; REGO, F. G. M.; PICHETH, G. COVID-19 e Diabetes: a relação entre duas pandemias distintas. Revista Brasileira de Análises Clínicas. DOI: 10.21877/2448-3877.20200001. 2020.

ASKIN, L.; TANRIVERDI, O.; ASKIN, H. S. The Effect of Coronavirus Disease 2019 on Cardiovascular Diseases. Arq. Bras. Cardiol., São Paulo, v. 114, n. 5, p. 817-822, mai. 2020.

ASOKAN, I.; RABADIA, S. V.; YANG, E. H. The COVID-19 Pandemic and its Impact on the Cardio-Oncology Population. Curr Oncol Rep., v. 22, n. 6, mai. 2020.

BIANCHETTI, A. et al. Clinical presentation of COVID-19 in dementia patients. J Nutr Health Aging, mai., 2020. DOI: 10.1007/s12603-020-1389-1.

BORNSTEIN, S. R. et al. Practical recommendations for the management of diabetes in patients with COVID-19. The lancet Diabetes \& endocrinology, v. 9, n. 6, p. 546550, jun. 2020.

BRASIL. Plano de contingência DATASUS: Situação de crise provocada pelo novo coronavírus (covid-19). Versão 1.0, 2020a.

BRASIL. MINISTÉRIO DA SAÚDE. Alzheimer: o que é, causas, sintomas, tratamento, diagnóstico e prevenção. Disponível em:< https://saude.gov.br/saudede-a-z/alzheimer>. Acesso em: 31 jul. $2020 \mathrm{~b}$.

BRASIL. MINISTÉRIO DA SAÚDE. Painel de casos de doença pelo coronavírus 2019 (COVID-19) no Brasil pelo Ministério da Saúde. Versão 2.0. Disponível em:< https://covid.saude.gov.br/>. Acesso em: 10 de jul. de 2020c. 
BUSSALINO, E. et al. Immunosuppressive therapy maintenance in a kidney transplant recipient SARS-CoV-2 pneumonia: a case report. Am J Transplant., abr. 2020. DOI: 10.1111/ajt.15920.

CAPARRO-GONZALEZ, R. A. COVID-19 in PregnantWomen and Neonates: A Systematic Review of the Literature with Quality Assessment of the Studies. Rev Esp Salud Pública., v. 94, abr. 2020.

CHENG, $Y$ et al. Kidney disease is associated with in-hospital death of patients with COVID-19. Kidney Internacional, v. 97, n. 5, p. 829-838, mar. 2020.

CHINAZZI, M. et al. The effect of travel restrictions on the spread of the 2019 novel coronavirus (COVID-19) outbreak. Science, v. 368, n. 6489, p. 395-400, mar. 2020.

COSTA, I. B. S. S. et al. O Coração e a COVID-19: O que o Cardiologista Precisa Saber. Arq. Bras. Cardiol., v. 114, n. 5, p. 805-816, mai. 2020a.

COSTA, F. F. et al. Metabolic syndrome and COVID-19: An update on the associated comorbidities and proposed therapies. Diabetes \& Metabolic Syndrome: Clinical Research \& Reviews, v. 14, n. 5, p. 809-814, 2020 b.

DIAS, N. L. et al. Predição da propagação do SARS-CoV-2 no Estado do Amapá, Amazônia, Brasil, por modelagem matemática. Revista Científica Multidisciplinar Núcleo do Conhecimento, vol. 06, ano 05, ed. 05, p 73-95, mai., 2020.

DRIGGIN, E. et al. Cardiovascular Considerations for Patients, Health Care Workers, and Health Systems During the COVID-19 Pandemic. J Am Coll Cardiol., v. 75, n. 18, p. 2352-2371, mai. 2020.

ENGIN, A. B.; ENGIN, E. D.; ENGIN, A. Two important controversial risk factors in SARS-CoV-2 infection: obesity and smoking. Environmental Toxicology and Pharmacology, v. 78, mai. 2020. DOI: 10.1016/j.etap.2020.103411. 
ENGSTROM, E. et al. Recomendações para a organização da Atenção Primária à Saúde no SUS no enfrentamento da Covid-19. Observatório Covid-19 Fiocruz, Nota técnica. p. 13, mai. 2020.

EHRMANN, J.; URBAN, O.; DVORAN, P. Alcohol-related liver diseases. Cent Eur J Public Health, v. 27, p. 10-14, dez. 2019.

FAN, C. et al. ACE2 Expression in Kidney and Testis May Cause Kidney and Testis Damage After 2019-nCoV Infection. MedRxiv, fev. 2020. DOI: 10.1101/2020.02.12.20022418.

FANG, L.; KARAKIULAKIS, G.; ROTH, M. Are patients with hypertension and diabetes mellitus at increased risk for COVID-19 infection?. The Lancet. Respiratory Medicine, v. 8, n. 4, mai. 2020.

FERINI-STRAMBI, L.; SALSONE, M. COVID-19 and neurological disorders: are neurodegenerative or neuroimmunological diseases more vulnerable?. J Neurol., jul. 2020. DOI: $10.1007 / s 00415-020-10070-8$.

FERRARI, F. COVID-19: Dados Atualizados e sua Relação Com o Sistema Cardiovascular. Arq. Bras. Cardiol., v. 114, n. 5, p. 823-826, mai. 2020. DOI: 10.36660/abc.20200215.

GAUTRET, P. et al. Hydroxychloroquine and azithromycin as a treatment of COVID19: results of an open-label non-randomized clinical trial. International journal of antimicrobial agents, mar. 2020. DOI: 10.1016/j.ijantimicag.2020.105949.

GIMENEZ, V. M. M. et al. Lungs as target of COVID-19 infection: Protective common molecular mechanisms of vitamin $D$ and melatonin as a new potential synergistic treatment. Life Sci., v. 354, ago. 2020. DOI: 10.1016/j.Ifs.2020.117808.

GUAN, W. et al. Clinical characteristics of coronavirus disease 2019 in China. New England journal of medicine, v. 382, n. 18, p. 1708-1720, fev. 2020. 
HONG, H. et al. Clinical characteristics of novel coronavirus disease 2019 (COVID-19) in newborns, infants and children. Pediatrics and Neonatology, v. 61, n. 2, p. 131132, abr. 2020.

HRUSAK, O. et al. Flash survey on severe acute respiratory syndrome coronavirus-2 infections in paediatric patients on anticancer treatment. European Journal of Cancer, v. 132, p. 11-16, abr. 2020.

HUANG, C.; WANG, Y.; LI, X. Características clínicas de pacientes infectados com o novo coronavírus de 2019 em Wuhan, China. Lanceta, v. 395, p. 497-506, jan. 2020.

HUSSAIN, A. Obesity and mortality of COVID-19. Meta-analysis. Obes Res Clin Pract., jul. 2020. DOI: 10.1016/j.orcp.2020.07.002.

KHALIQ, O. P.; PHOSWA, W. N. Is Pregnancy a Risk Factor of COVID-19?. European Journal of Obstetrics \& Gynecology and Reproductive Biology, 2020. DOI: 10.1016/j.ejogrb.2020.06.058.

IACOBELLIS, G. COVID-19 and diabetes: can DPP4 inhibition play a role?. Diabetes research and clinical practice, v. 162, abr. 2020. DOI: 10.1016/j.diabres.2020.108125.

LI, H. et al. Coronavirus disease 2019 (COVID-19): current status and future perspective. International journal of antimicrobial agents, v. 55, n. 5, mai. 2020.

LIU, H. et al. Why are pregnant women susceptible to COVID-19? An immunological Viewpoint. Journal of reproductive immunology, v. 139, jun. 2020.

LIMA, D. L. P. et al. COVID-19 no estado do Ceará, Brasil: comportamentos e crenças na chegada da pandemia. Ciênc. saúde coletiva, v. 25, n. 5, p. 1575-1586, mai. 2020.

LUCENA, T. M. C. et al. Mechanism of inflammatory response in associated comorbidities in COVID-19. Diabetes \& Metabolic Syndrome: Clinical Research \& Reviews, mai. 2020. DOI: 10.1016/j.dsx.2020.05.025. 
MA, R. C. W.; HOLT, R. I. G. COVID-19 and diabetes. Diabetic Medicine, abr. 2020. DOI: $10.1111 / \mathrm{dme} .14300$.

MARTINS-CHAVES, R. R.; GOMES, C. C.; GOMEZ, R. S. Immunocompromised patients and coronavirus disease 2019: a review and recommendations for dental health care. Braz. res oral., São Paulo, v. 34, e048, mai. 2020.

MENDONÇA, F. D. Região Norte do Brasil e a pandemia de COVID-19: análise socioeconômica e epidemiológica. Journal Health NPEPS, v. 5, n. 1, 2020.

MOITINHO, M. S. et al. Lesão renal aguda pelo vírus SARS-COV-2 em pacientes com COVID-19: revisão integrativa. Rev. Bras. Enferm., Brasília, v. 73, n. 2, jul. 2020.

OLIVEIRA, A. C.; LUCAS, T. C.; IQUIAPAZA, R. A. What has the COVID-19 pandemic taught us about adopting preventive measures?. Texto \& Contexto Enfermagem, Florianópolis, v. 29, mai. 2020. DOI: 10.1590/1980-265x-tce-2020-0106.

OLIVEIRA, W. K. et al. Como o Brasil pode deter a COVID-19. Epidemiol. Serv. Saude, Brasília, v. 9, n. 2, p. 1-8, abr. 2020.

OMS. ORGANIZAÇÃO MUDIAL DA SAÚDE. Painel da Doença de Coronavírus da OMS (COVID-19). Disponível em:< https://covid19.who.int/>. Acesso em: 10 de jul. de 2020.

OYELADE, T.; ALQAHTANI, J.; CANCIANI, G. Prognosis of COVID-19 in Patients with Liver and Kidney Diseases: An Early Systematic Review and Meta-Analysis. Trop. Med. Infect. Dis., v. 5, n. 80, mai. 2020.

PRANATA, R. et al. Impact of cerebrovascular and cardiovascular diseases on mortality and severity of COVID-19-systematic review, meta-analysis, and metaregression. J Stroke Cerebrovasc Dis., v. 29, n. 8,: mai. 2020. 
PIRES, L. N.; CARVALHO, L.; XAVIER, L. L. COVID-19 e desigualdade: a distribuição dos fatores de risco no Brasil. Research Gate, abr. 2020. DOI: 10.13140/RG.2.2.27014.73282.

RAJKUMAR, R. P. Ayurveda and COVID-19: Where psychoneuroimmunology and the meaning response meet. Brain, behavior and immunity, v. 87, p. 8-9, jul. 2020.

RENU, K.; PRASANNA, P. L.; GOPALAKRISHNAN, A. V. Coronaviruses pathogenesis, comorbidities and multi-organ damage - A review. Life sci., v. 255, mai. 2020. DOI: 10.1016/j.lfs.2020.117839.

RODRÍGUEZ-COLA, M. et al. Clinical features of coronavirus disease 2019 (COVID19) in a cohort of patients with disability due to spinal cord injury. Spinal Cord Series and Cases, v. 6, n. 39, mai. 2020.

RONCON, L. et al. Diabetic patients with COVID-19 infection are at higher risk of ICU admission and poor short-term outcome. Journal of Clinical Virology, v. 127, jun. 2020. DOI: 10.1016 / j.jcv.2020.104354.

RUBIO-PÉREZ, I. et al. COVID-19: Conceptos clave para el cirujano. Cirugía Española, v. 98, n. 6, p. 310-319, jun./jul. 2020.

SIA, S. F. et al. Pathogenesis and transmission of SARS-CoV-2 in golden hamsters. Nature, mai. 2020. DOI: 10.1038/s41586-020-2342-5.

SILVA, A.W.C. et al. Caracterização clínica e epidemiologia de 1560 casos de COVID19 em Macapá/AP, extremo norte do Brasil. Research, Society and Development, v. 9, n.8, e150985499, jun. 2020.

STEFAN. N. et al. Obesity and impaired metabolic health in patients with COVID-19. Nat Rev Endocrinol., abr. 2020. DOI: 10.1038/s41574-020-0364-6.

TAY, M. Z. et al. The trinity of COVID-19: immunity, inflammation and intervention. Nat Rev Immunol, v. 20, p. 363-374, abr. 2020. DOI: 10.1038/s41577-020-0311-8. 
TADIC, M. et al. COVID-19 and arterial hypertension: Hypothesis or evidence? J Clin Hypertens (Greenwich), jul. 2020. DOI:10.1111/jch.13925.

TRIPPELA, G. et al. COVID-19 in PregnantWomen and Neonates: A Systematic Review of the Literature with Quality Assessment of the Studies. Pathogens, v. 9, n. 6, jun. 2020.

TROYER, E. A.; KOHN, J. N.; HONG, S. Are we facing a crashing wave of neuropsychiatric sequelae of COVID-19? Neuropsychiatric symptoms and potential immunologic mechanisms. Brain, behavior and immunity, v. 87, p. 34-39, jul. 2020.

TUTIYA, C. T. et al. Possible formation of pulmonary microthrombi in the early puerperium of pregnant women critically ill with COVID-19: Two case reports. Case Rep Womens Health, v. 27, e00237, jun. 2020.

VARDAVAS, C. I.; NIKITARA, K. COVID-19 and smoking: A systematic review of the evidence. Tob Induc Dis., v. 18, mar. 2020. DOI: 10.18332/tid/119324.

WANG, D. et al. Clinical Characteristics of 138 Hospitalized Patients With 2019 Novel Coronavirus-Infected Pneumonia in Wuhan, China. Journal of American Medical Asssociation, v. 323, n. 11, p. 1061-1069, fev. 2020.

YANG, J. et al. Prevalence of comorbidities and its effects in patients infected with SARS-CoV-2: a systematic review and meta-analysis. Int $\mathbf{J}$ Infect Dis., v. 94, p. 9195, mai. 2020.

YAO, H.; CHEN, J. H.; XU, Y. F. Patients with mental health disorders in the COVID19 epidemic. Lancet Psychiatry, v. 7, n. 4, mar. 2020.

ZHAI, P. et al. The epidemiology, diagnosis and treatment of COVID-19. International journal of antimicrobial agents, v. 55, n. 5, mai. 2020. 
ZHANG, L. et al. Clinical characteristics of COVID-19-infected cancer patients: a retrospective case study in three hospitals within Wuhan, China. Ann Oncol., v. 31, n. 7, p 894-901, jul. 2020.

Представлено: Июнь 2021.

Утверждено: Июнь 2021. 